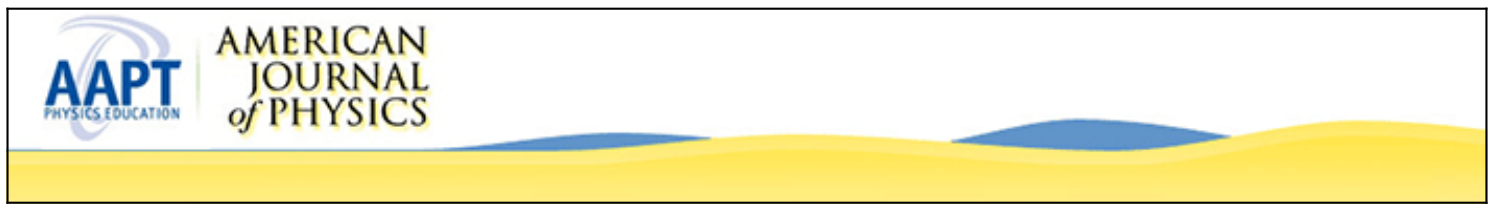

\title{
Proportional Counters for Demonstration Experiments
}

A. Moljk and R. W. P. Drever

Citation: American Journal of Physics 25, 165 (1957); doi: 10.1119/1.1934387

View online: http://dx.doi.org/10.1119/1.1934387

View Table of Contents: http://scitation.aip.org/content/aapt/journal/ajp/25/3?ver=pdfcov

Published by the American Association of Physics Teachers

\section{Articles you may be interested in}

Proportional Counter

Am. J. Phys. 32, xix (1964); 10.1119/1.1970214

Proportional Counter

Am. J. Phys. 30, xiii (1962); 10.1119/1.1941881

The Scintillation Counter as a Proportional Device

Rev. Sci. Instrum. 20, 178 (1949); 10.1063/1.1741484

Air Proportional Counters

Rev. Sci. Instrum. 19, 733 (1948); 10.1063/1.1741155

Operation of Proportional Counters

Rev. Sci. Instrum. 12, 94 (1941); 10.1063/1.1769837

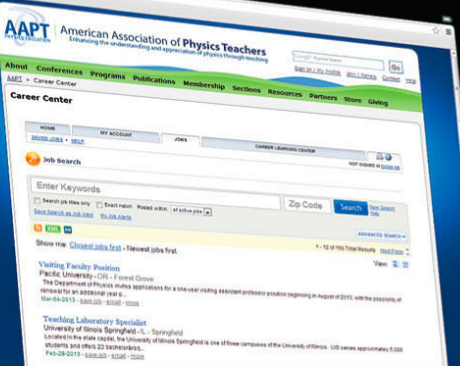

American Association of Physics Teachers

Explore the AAPT Career Center -

access hundreds of physics education and

other STEM teaching jobs at two-year and

four-year colleges and universities.

\section{http://jobs.aapt.org}




\title{
Proportional Counters for Demonstration Experiments
}

\author{
A. Moljk* and R. W. P. Drever \\ Department of Natural Philosophy, University of Glasgow, Glasgow, Scotland
}

(Received June 4, 1956)

\begin{abstract}
Proportional counters suitable for demonstration purposes are described, and some experiments to illustrate counter operation are discussed. The charge carried by the positive ions produced in the multiplication process, and the localization of the avalanche, is revealed in a special proportional counter whose cathode is covered by a layer of polythene. Other properties of this type of counter, such as its ability to work for a considerable period of time without a high-voltage supply, are pointed out.
\end{abstract}

$T$ HE phenomena studied in nuclear physics are still far from everyday experience, so demonstration experiments are particularly important in this field. For demonstrations on radioactivity and $\mathrm{x}$-rays a proportional counter ${ }^{1}$ is a very useful instrument, and the properties and operation of the counter itself are also well worth showing.

Proportional counters may be obtained commercially, but a counter such as that shown in Fig. 1 is easily constructed and is more suitable for demonstration purposes since the internal design is plainly apparent. A brass tube is fitted at both ends with Lucite stoppers, sealed with wax or araldite. A steel wire, $4 \mathrm{mil}$ in diameter, is mounted along the axis and is kept under tension by a spring at one end. The wire is brought out at the other end through a small

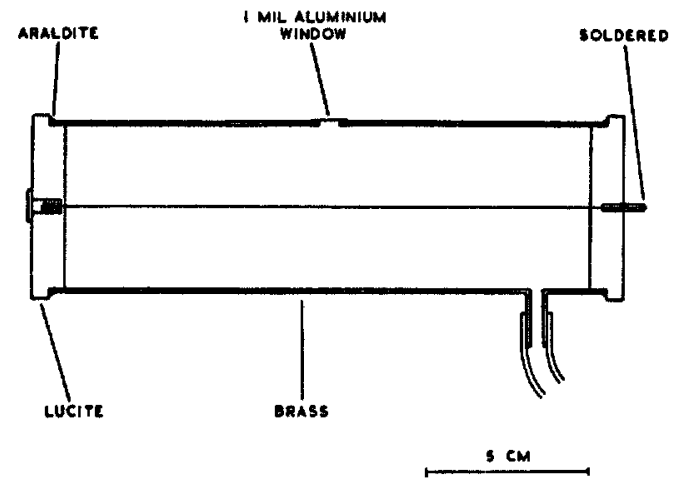

FIg. 1. A simple proportional counter suitable for demonstration experiments.

* On leave from the University of Ljubljana, Yugoslavia.

1 Two recent review articles on proportional counters are: S. C. Curran, "Proportional counter spectrometry" in Beta- and Gamma-Ray Spectroscopy, edited by K. Seigbahn (North Holland Publishing Company, Amsterdam, 1955); D. West, Progr. Nuclear Phys. 3, 18 (1953).

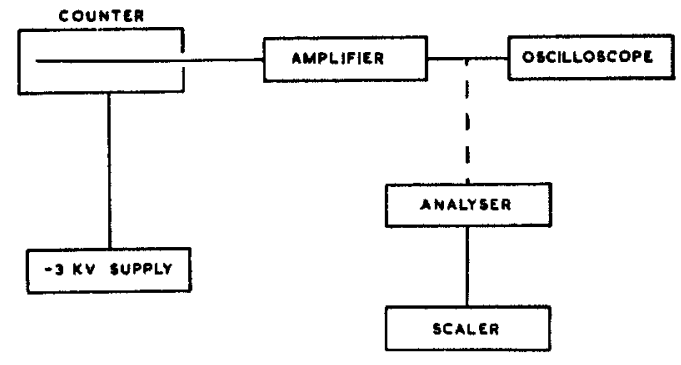

FIG. 2. Schematic diagram of equipment for demonstrating the counters.

metal tube which serves as the external connection. To make the counter suitable for the detection of beta particles and soft $\mathrm{x}$-rays a $\frac{1}{4}$ in. hole is bored in the side and covered with thin aluminium foil cemented with araldite. The counter is evacuated with a rotary pump, flushed with argon, and filled with methane and argon to partial pressures of 10 and $70 \mathrm{~cm}$ of mercury, respectively.

To operate the counter a potential difference of about $2.5 \mathrm{kv}$ must be applied between the wire and the case. The usual arrangement is shown in Fig. 2. The case is connected to the negative terminal of a stabilized high-voltage supply so that the wire can be connected directly to the input of a linear amplifier. The gain required is about 30000 , and differentiation and integration time constants of about $15 \mu \mathrm{s}$ are suitable. A simple oscilloscope with a time base frequency of $50 \mathrm{cps}$ is sufficient to display the pulses.

The use of an amplifier and an oscilloscope should not make the experiment incomprehensible. Nowadays we are accustomed to electronic instruments and we can understand how the 
counter operates without knowing the internal design of the amplifier or power unit, just as we can perform experiments in mechanics without knowing how a stopwatch is made. It is sufficient to understand the purpose of each instrument and how it is used.

To demonstrate the characteristics of a proportional counter a source of monoenergetic electrons is desirable. A beta emitter is not entirely suitable because of the continuous spectrum. However the photoelectrons produced in the counter gas when monochromatic $x$-rays are passed through it are equivalent to monoenergetic beta particles. Such $\mathrm{x}$-rays may be conveniently obtained from radioactive materials which decay by $K$ capture, and for our purpose germanium-71 is a very suitable source. It decays with a half-life of 11.4 days and emits the $9.2 \mathrm{kev}$ characteristic $\mathrm{x}$-rays of gallium. Germanium-71 is supplied in the form of $\mathrm{GeO}_{2}$ powder, and about $100 \mu \mathrm{c}$ of the source enclosed in Lucite is sufficient. When the germanium source is placed above the aluminum window, and the voltage on the counter is set at about $2.2 \mathrm{kv}$, numerous pulses of approximately the same height appear on the oscilloscope. If the voltage is increased the pulses become larger, due to the increase of gas multiplication in the counter. By using a source which emits $x$-rays of another energy it can be shown that the pulse height is proportional to the energy of absorbed photons. A suitable source is iron-55 which gives $\mathrm{x}$-rays of $6 \mathrm{kev}$.

The proportionality between pulse height and energy can also be demonstrated by using the fluorescence radiation from materials such as copper and silver, excited by the beam from an $\mathrm{x}$-ray machine (see Fig. 3). A piece of metal foil is mounted, inclined at $45^{\circ}$, above the window of

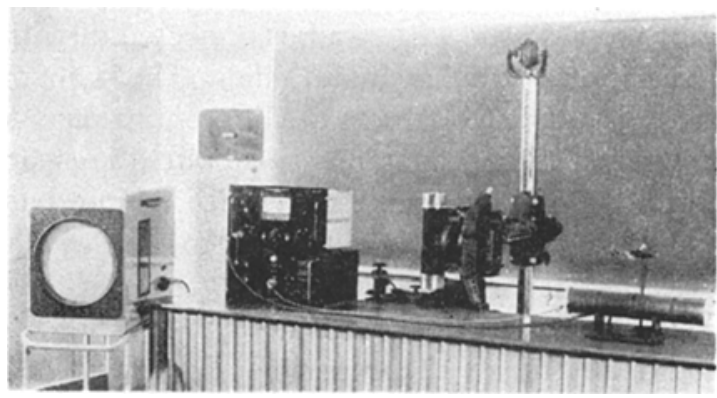

FIG. 3. Arrangement for showing the fluorescence radiation excited by $x$-rays in copper. the counter and a narrow beam of $x$-rays from a small $x$-ray set is directed horizontally onto the foil. If the counter is shielded from direct $\mathrm{x}$-rays from the machine, only pulses corresponding to the $K$ radiation of the target foil will be seen on the oscilloscope. For copper the energy of $K$ radiation is $8.0 \mathrm{kev}$ and for silver $22.2 \mathrm{kev}$ and the heights of pulses observed with these foils will be proportional to the energies. If a pulse-height analyzer is available the experiment can be made more accurate and energy resolution can also be readily measured.

The above experiments illustrate the characteristics of a normal proportional counter as an energy measuring instrument. Its use as a mere detector of nuclear radiation can be shown by many other straightforward experiments with $\alpha, \beta$, and $\gamma$ sources. However in order to demonstrate some aspects of the internal mechanism of counter action a special type of proportional counter has been developed and is described below.

At this point it may be worthwhile to give a brief account of the multiplication process in a proportional counter.

A primary particle passing through the counter ionizes the gas, losing about $30 \mathrm{ev}$ of its energy for each ion pair produced. Under the influence of the electric field, the secondary electrons drift towards the wire into a region of rapidly increasing field strength. Close to the wire the field is high enough to give each electron sufficient energy to ionize a gas atom. 'The two free electrons now present both ionize at their next collisions, and the effect is cumulative. If the multiplication starts at a distance of $n$ mean free paths from the wire the total gain is $2^{n}$. Since the mean free path is of the order of $10^{-3}$ $\mathrm{cm}$ the whole process occurs within a few tenths of a $\mathrm{mm}$ of the wire. When the voltage is increased the avalanche starts further from the wire and the multiplication is higher. The avalanche is localized to that part of the wire immediately below the primary track, which is not the case in a Geiger counter where the discharge spreads along the whole wire.

The electrons produced in the avalanche are collected by the wire in a microsecond or less and give rise to the initial part of the output pulse. However the major contribution to the 


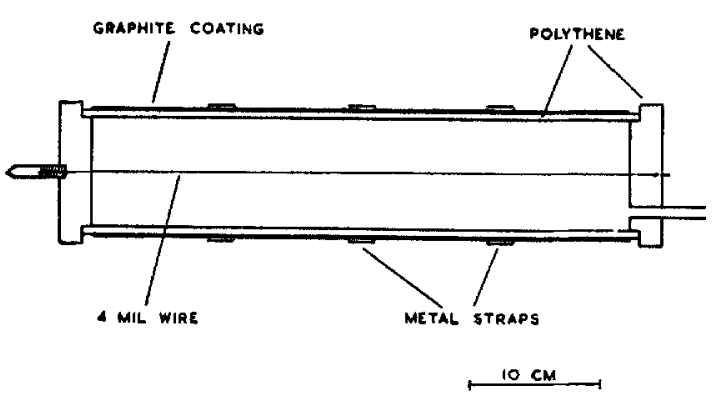

Fig. 4. An external cathode proportional counter made of polythene.

pulse comes from the relatively slow movement of the positive ions, from the avalanche, through the high field region surrounding the wire. These ions are eventually neutralized at the cathode.

The charge carried by the positive ions, and the localization of the avalanche, can both be shown by using a counter whose cathode is covered with a layer of good insulating material. Such a counter is shown in Fig. 4. It is made almost entirely from polythene, so it is very easy to construct. However, other plastics can also be used. The case is a $\frac{1}{4}$-in. thick polythene tube welded to endpieces turned from 1 in. polythene sheet. The outside of the tube is given a conducting coating of graphite, which forms the cathode, and three metal straps are fitted around it to prevent collapse when pumped out. An aluminum window is not required in this counter since soft $\mathbf{x}$-rays are not appreciably absorbed in the polythene which consists only of elements with low atomic number.

The wire is brought through small holes in the end-plates and is kept tight by a spring enclosed in a piece of polythene tubing. It may be remarked that for our purpose the polythene parts can be easily sealed together by dropping molten material on to the joint from a piece of burning polythene. The counter is evacuated and filled with the usual argon-methane mixture mentioned above. Since the polythene is difficult to degas the filling deteriorates with time, but the counter can be used for about a week without refilling.

The counter is connected to a power unit and amplifier in the normal way, and when a germanium source is in the vicinity, pulses of uniform height are seen on the cathode-ray tube just as with a conventional counter. This might be expected since the presence of a relatively thin layer of dielectric material on the cathode, where the electric field is weak, does not change the field distribution in the gas appreciably.

However, after a few minutes of operation it will be noticed that the pulse height decreases. This is due to a reduction in the field inside the counter caused by the accumulation of charge, brought by the positive ions, on the surface of the polythene.

This charge can be removed and the counter restored to its original condition by irradiation with the beam from an $x$-ray set, with the highvoltage supply to the counter switched off. If a self-rectifying $x$-ray set is used the discharge current is modulated at mains frequency and can be seen on the oscilloscope.

The magnitude of the charging-up effect can be readily calculated for the case when the whole counter is uniformly irradiated. The polythene layer may be regarded as a condenser of capacity $C$ in series with the actual counter. When a primary particle loses energy $u$ in the gas of the counter, $u(A+1) / W$ ion pairs are produced, where $A$ is the gas multiplication and $W$ is the energy per ion pair. The arrival of the positive ions on the polythene surface from $N$ such primary particles causes a drop in voltage across the counter of $N u(A+1) e / W C$, $e$ being the electron charge, and there is a corresponding fall in gain.

This expression can be verified experimentally by observing the times required for a given change of pulse height with different values of gas multiplication and source strength. The absolute value of the gas multiplication at a certain voltage can also be obtained in this way.

In order to show that the avalanche in a proportional counter is localized, the germanium source is used to irradiate a small area of the counter until the pulse height decreases considerably. If the source is then moved along the axis of the counter the change of gain will be found to have occurred only in the region that was irradiated.

The experiments described above with the polythene counter show that some aspects of gas multiplication can be illustrated with this counter more directly than with a normal one. 
However, in addition to being useful for demonstration purposes, this counter has further interesting properties.

The polythene counter can be made to operate for a considerable period of time without any high-voltage supply. The counter must first be irradiated by an $\mathrm{x}$-ray machine while a voltage is applied equal to the normal operating voltage, but reversed in sign so that the case is positive. When the charging process is complete, and the high voltage is switched off, the counter will operate as usual due to the negative charge on the inner surface of the polythene. The pulse height decreases slowly, the rate of decrease depending on the gain in the counter, the energy and intensity of radiation. In the counter shown in Fig. 4 the gain fell from 10000 to 2000 in $12 \mathrm{hr}$ exposure to the natural cosmic ray background of 450 counts per minute.

The counter may be used as an integrating instrument for the measurement of total radiation over a certäin time, by observing the fall in gain that occurs. Some information about the distribution of ionization along the counter may also be obtained, and in this respect the counter may be said to have a "memory" for radiation.

\title{
Physics and Physical Science in New Orleans, 1800-1860
}

\author{
KARLEM RIESS \\ Tulane University, New Orleans, Louisiana
}

(Received July 16, 1956)

\begin{abstract}
A survey of the early developments in physical science in New Orleans, with emphasis on the important contributions of John Leonard Riddell, Edward Hall Barton, Claudius Wistar Sears, and Caleb G. Forshey.
\end{abstract}

$S^{c}$ CIENTIFIC thinking in New Orleans in the early years of the past century was primarily associated with three groups - traveling lecturers, local physicians, and a few faculty members of the University of Louisiana. During this period New Orleans was the center of a rich, agricultural region, and a busy port. There was much wealth and splendor, but also gambling, corruption, filth, and violence. Stabbings, garrotings, and duels were quite common. There was no sanitary water supply, most of the residents relying on rain water collected in cisterns. There was no sewerage system and refuse was allowed to accumulate in the mud streets. Characteristically, the native-born residents were quite content with their city, and no effort was made to correct these matters. Only visitors and those moving to New Orleans from other areas were concerned.

In spite of these shortcomings there was a genuine interest in things scientific. Three medical and scientific societies were organized before 1840. A museum was opened in 1819 , another in 1825 , and a third in 1836 . There were organized discussion groups, meeting informally, for the exchange of ideas and experiences. It is not surprising that most of these were concerned with natural history or with medicine, since the region abounded in all sorts of specimens, and was visited periodically by epidemics of yellow fever and cholera.

The influence of the traveling lecturer was considerable. These men were not charlatans, but usually scientists of established reputation, who gave short courses of lectures. The earliest visitor of this type arrived in 1819, and gave a series on "Chemical and Physical Enquiries." There were lectures on microscopes and telescopes, courses in astronomy, geology, chemistry, meteorology, and natural philosophy, and in applied science. Dionysius Lardner, the Irish scientist, drew a large and interested audience for his lectures on physical and astronomical subjects in 1843. Benjamin Silliman gave his lectures on geology in 1845, and Benjamin Silliman, Jr. presented a course entitled the "Chemistry of Agriculture." The audience re- 